\title{
Depression of Synaptic Efficacy at Intermolt in Crayfish Neuromuscular Junctions by 20-Hydroxyecdysone, a Molting Hormone
}

\author{
ROBIN L. COOPER AND MARVIN E. RUFFNER \\ Nerve-Muscle Group, Thomas Hunt Morgan School of Biological Sciences, University of Kentucky, Lexington, \\ Kentucky 40506-0225
}

\begin{abstract}
Cooper, Robin L. and Marvin E. Ruffner. Depression of synaptic efficacy at intermolt in crayfish neuromuscular junctions by 20 hydroxyecdysone, a molting hormone. J. Neurophysiol. 79: 19311941, 1998. This report demonstrates that ecdysteroids can reduce synaptic transmission at an intermolt stage of a crustacean tonic neuromuscular junction by acting at a presynaptic site. The steroid molting hormone, 20-hydroxyecdysone (20-HE), appears to act through a rapid, nongenomic mechanism that decreases the probability of synaptic vesicle release and reduces the number of release sites. Quantal analysis revealed that fewer vesicles were released for a given stimulus when 20-HE was present, and this in turn accounted for the reduced synaptic efficacy. Reduced synaptic efficacy produced smaller evoked postsynaptic currents and smaller excitatory postsynaptic potentials (EPSPs) across the muscle fiber membrane. The reduction in EPSPs was observed among muscle fibers that were innervated by high- or low-output terminals. The behavior of crustaceans/crayfish during the molt cycle, when 20$\mathrm{HE}$ is high, may be explained by the reduction in synaptic transmission. Crustaceans become quiescent during the premolt periods as do insects. The effects of 20-HE can be reversed with the application of the crustacean neuromodulator serotonin, which enhances synaptic transmission.
\end{abstract}

\section{INTRODUCTION}

During molting, a crustacean sheds its exoskeleton and replaces it with one large enough in which to grow. The ecdysteroid hormones play an important role in directing the preparation of the animal for the premolt and molt. To characterize the restructuring of the motor neurons and muscles during metamorphosis, extensive experimental use has been made of members of another arthropod subphylum, Insecta: Drosophila, and the tobacco hornworm, Manduca sexta, in particular. In Manduca, some motor neurons undergo sprouting of their dendritic trees and others exhibit regression of dendritic trees and some motor neurons even undergo cell death under the influence of ecdysteroids (Jacobs and Weeks 1990; Levine and Weeks 1996; Levine et al. 1986; Truman 1996; Truman and Reiss 1995). Drosophila demonstrates ecdysteroid-dependent regulation of gene expression (Segraves 1994; Thummel 1996) by the now-familiar mechanism of steroid-dependent receptor binding to response elements on genomic DNA. Transcriptional activation of early-response genes results in modulation of expression of other genes (Thummel 1996; White et al. 1997). To date, all characterized ecdysteroid actions in insects indicate a genomic effect. In addition to the generalized genomic effects of estradiol, aldosterone, vitamin $\mathrm{D}_{3}$, and cortisol in mammalian systems, there is now substantial documentation that nongenomic, membrane-bound steroid receptors can mediate rapid action of cellular processes such as inositol tri-phosphate $\left(\mathrm{IP}_{3}\right)$ release, increased flux of calcium from internal stores, higher levels of guanosine $3^{\prime}, 5^{\prime}$-cyclic monophosphate (cGMP) and adenosine 3',5'-cyclic monophosphate (cAMP), and direct activation of a $\mathrm{Na}^{+} / \mathrm{H}^{+}$antiporter (Wehling 1995).

Studies investigating the nongenomic actions of molt-related compounds among crustaceans and insects are sparse. Behavioral changes occur in crustaceans in preparation for and immediately after molting, but these mechanisms are not fully understood. There is an indication that the moltrelated hormone may induce the behaviors associated with this period (Cromarty 1995). The present study shows that in neuromuscular preparations in which the axon had been severed from its cell body, ecdysone elicited a rapid response. The rate at which this response occurred suggested a direct action without transcriptional regulation within the presynaptic terminal or possibly in the muscle fibers.

On exposure of an isolated crayfish nerve-muscle preparation to 20-hydroxyecdysone (20-HE) there was a pronounced reduction in the size of the excitatory postsynaptic potentials (EPSPs) recorded in the muscle. To determine if 20-HE caused differential release of the neurotransmitter, evoked as well as spontaneous synaptic currents were recorded for subsequent quantal analysis of synaptic efficacy. Quantal analysis allowed assessment of changes caused by addition of particular neuromodulators to the electrophysiological characteristics of both pre- and postsynaptic cells. For example, a difference in the rate of spontaneous release or in latency jitter of an evoked release suggested a presynaptic mechanism of action, whereas a difference in the size of the response to release of a single vesicle indicated a postsynaptic role.

\section{METHODS}

\section{Animals}

All experiments were performed using the first and second walking legs of crayfish, Procambarus clarkii, measuring $6-10 \mathrm{~cm}$ in body length (Atchafalaya Biological Supply, Raceland, LA). Animals were housed in an aquatic facility and fed an ample supply of fish food and carrot pellets. Crayfish were induced to autotomize the walking leg of choice by forcefully pinching at the merus segment with a pair of forceps. The opener muscle of the first walking legs was prepared via standard dissection (Dudel and 
Kuffler 1961). The tissue was positioned in a silicone elastomer (Sylgard) dish for viewing with a Nikon Optiphot-2 epifluorescence microscope with a $\times 40$ ( 0.55 NA) Nikon water-immersion objective. Dissected preparations were maintained in crayfish saline, a modified Van Harreveld's solution [containing (in $\mathrm{mM}$ ) $205 \mathrm{NaCl}, 5.3 \mathrm{KCl}, 13.5 \mathrm{CaCl}_{2} \cdot 2 \mathrm{H}_{2} \mathrm{O}, 2.45 \cdot \mathrm{MgCl}_{2} 6 \mathrm{H}_{2} \mathrm{O}$, and $0.5 \mathrm{~N}$-2-hydroxyethylpiperazine- $N^{\prime}$-2-ethanesulfonic acid ] at $14^{\circ} \mathrm{C}$ and adjusted to $\mathrm{pH} 7.4$.

20-HE (Sigma), the active form of ecdysone in crustaceans, was dissolved freshly on the day of experimentation to a concentration of $10 \mu \mathrm{M}$ in crayfish saline. The entire bathing solution was exchanged two times within 1 min with the 20-HE containing saline. Serotonin (5-HT; Sigma) was diluted freshly from a frozen stock before each experiment. Both solutions were photosensitive and were stored in the dark until used. Extraneous light was blocked by drapes around the Faraday cage, and, after placing the solutions in the recording chamber on the microscope, the illuminating lamp was turned off.

\section{Instrumentation}

Intracellular recordings were performed with 30-60 $\mathrm{M} \Omega$ resistance microelectrodes filled with $3 \mathrm{M} \mathrm{KCl}$. Responses were recorded with a $1 \times$ LU headstage HS2A (Axon Instruments) and an Axoclamp 2A amplifier. Focal macropatch recordings were made with 10- to $12-\mu \mathrm{m}$ diam, fire-polished, glass electrodes placed directly over the nerve terminals. To record synaptic currents, a $0.1 \times$ LU head stage was used. Electrical signals were recorded to VHS tape (Vetter, 400), as well as on-line to a Power Mac 9500, via a MacLab/4 s interface. The EPSPs were recorded at 10 $\mathrm{kHz}$ with an intracellular electrode. The focal current recordings were recorded at either 10 or $20 \mathrm{kHz}$ with a focal macropatch electrode. All events were scaled appropriately to a known current test pulse applied through the patch electrode. The corrected scale then was adjusted with MacLab Scope software (version 3.5.4). The seal resistances were in the range of $100 \mathrm{~K} \Omega$ to $1 \mathrm{M} \Omega$ and were monitored throughout the recording period using test current pulses. If there was any change noted throughout the experiment, the amplitude and area of the excitatory postsynaptic currents (EPSCs) and miniature excitatory postsynaptic currents (mEPSCs) were discarded. Stimulation to the opener nerve was obtained by use of a Grass S-88 simulator and a stimulation isolation unit (Grass, SIU) with leads to a standard suction electrode set-up (Cooper et al. 1995a).

\section{Anatomy}

The opener muscle is innervated by a single, tonic excitatory motor neuron. Dissection of the opener muscle consisted of removing the closer muscle and its associated cuticle. This allowed for a ventral view of the entire opener muscle. Differences in the branching pattern of the opener motor nerve terminals were observed. These differences depend on the region of the muscle innervated by the motor neuron. Specifically, the terminals form long strings with intermittent varicosities on the central muscle fibers, whereas the terminals are more highly branched on the proximal muscle fibers (Cooper et al. 1995a, 1996c). The terminals on the central muscle fibers have a substantially lower output in synaptic transmission when compared with the terminals on the proximal fibers. There are also structural differences in the synapses that, in part, account for the differences in the degree of synaptic efficacy (Cooper et al. 1996b,c).

\section{EPSPS}

EPSPs were recorded simultaneously in the proximal and central muscle fibers to determine if there were differential effects of
20-HE in these two distinct regions. Selective stimulation of the excitatory axon was carried out as described by Dudel and Kuffler (1961). The axon was stimulated with trains of 9 and 10 stimuli at 30,40 , and $50 \mathrm{~Hz}$ with a train interval of $10 \mathrm{~s}$. Averages of $10-$ 20 trains were used for each measurement. Facilitation $(\mathrm{Fe})$ was determined by measuring the peak amplitude of the corrected 10th EPSP, dividing it by the amplitude of the 1st EPSP, and subtracting one from this quotient: 10 th $/ 1$ st -1 . The 10th EPSP was corrected because its amplitude was offset because the response rides on the decay of the 9th pulse. To determine the time point during the 9th stimulus at which the peak of the 10th EPSP occurred, trains containing 9 stimuli were given intermittently among the trains of 10 stimuli, and the corresponding time point of the 10th EPSP peak was measured on the decay of the 9th EPSP. This offset value then was subtracted from the 10th EPSP amplitude. Because the resting membrane potential of the muscle fibers ranged from -75 to $-65 \mathrm{mV}$ and the EPSP amplitudes were not large enough to affect nonlinear summation, correction factors for nonlinear summation were not implemented for this study (Martin 1955). When nonlinear summation is corrected for in this type of preparation, as noted in others, there appears to be an overcompensation (Martin 1976).

After the 20-HE application, the preparations were rinsed with crayfish saline several times. In some cases, facilitation was remeasured during this washing period. After the wash, $1 \mu \mathrm{M}$ 5-HT was added, and the preparation was examined to see if it was as receptive to the 5-HT after a 20-HE application as before (Crider and Cooper 1996, 1997).

\section{Focal macropatch recordings}

The living nerve terminals were visualized with the vital fluorescent dye, 4-[4-(diethylamino) styryl]- $N$-methylpyridinium iodide (4-Di-2-Asp; Molecular Probes, Eugene, OR) (Magrassi et al. $1987)$ at $2-5 \mu \mathrm{M}$ in crayfish saline. The preparation was exposed to the dye solution for 2-5 min, followed by washing in crayfish saline. The synaptic transmission remained unaltered by this dye as previously shown (Cooper et al. 1995a). Selected terminal regions were photographed and traced to scale to determine the recorded terminal's location.

The synaptic current recordings were obtained using a loosepatch technique by lightly placing the $10-$ to $12-\mu \mathrm{m}$-diam lumen of the electrode directly over a single, spatially isolated varicosity along the vital dye-visualized nerve terminal. The macropatch electrode was quite specific for currents recorded at the patched site. The bathing fluid then was drawn into the lumen of the patch electrode. The seal resistance was maintained throughout the recording period as determined by applying test current pulses. The seal resistance among experiments was in the range of $100 \mathrm{~K} \Omega$ to $1 \mathrm{M} \Omega$. In each trace, a stimulus artifact and a nerve spike were observed, thus confirming nerve stimulation. Evoked, EPSCs and mEPSCs were analyzed to determine $m, n$, and $P$ as earlier described (Cooper et al. 1995b). In brief, direct counts of evoked events were made at a given continuous stimulation frequency throughout the recording. These direct counts provided one of the indices used to determine a mean quantal content $\left(m_{\mathrm{co}}\right)$. Direct counts of these evoked quantal events, the quantal parameters $n$ (number of release sites) and $P$ (probability of release at a site), were determined. In addition, the distribution in the number of releases was determined to be either a uniform binomial, a nonuniform binomial, or a Poisson distribution. The data sets were tested for a best-fit based on assumptions discussed in earlier reports (Dudel and Kuffler 1961; Wernig 1975). Binomial distributions previously have been shown to represent the quantal nature of release in crayfish neuromuscular junctions (Johnson and Wernig 1971; Wojtowicz et al. 1991). To test for nonuniform binomial 
distributions, the model and procedures described earlier in detail were used (Wojtowicz et al. 1991). The $\chi^{2}$ and a modified Akaike information criterion were used to estimate the distribution that best fitted events. A bootstrap method, as previously described in Cooper et al. (1995b), was employed to estimate the standard error of the estimated $n$ and $P$ values. The bootstrap method consisted of drawing 1,000 random samples from the original data set based on an open scheme (Efron and Tibshirani 1993).

There appeared to be gradual changes in all the quantal parameters on the addition of 20-HE. The sample sets of data for every 200 events were sufficient to obtain statistically significant values for quantal predictions in lieu of using the grouped total of 1,000 events. The 1,000 sampled, grouped data and the 200 event-group sets were compared. (For the convenience of the reader, the tabulated data analysis of only the 1,000 sampled sets are given ). Mean quantal content, determined by two other approaches, also was implemented for all the synaptic current recordings. Measurements of the maximum peak of each evoked event, including failures, provided an average evoked peak. This value then was divided by the mean peak amplitude of spontaneous events to provide the mean quantal content determined by the peak amplitude $\left(m_{\mathrm{pk}}\right)$ approach (Del Castillo and Katz 1954). It should be noted that the time to peak of evoked events varied, thus the point in time in which the measurements were made was shifted to obtain the true peak of each evoked response. The area under the trace or charge $(\mathrm{pA} \times \mathrm{ms})$ of the evoked events and failures similarly was divided by the mean charge of the spontaneous events to provide the mean quantal content $\left(m_{\mathrm{ch}}\right)$ by the charge approach. The window for integration of the evoked currents was determined by examining the time in which the averaged evoked current returned to baseline. This time point was used to scan through the evoked events to ascertain if any late evoked responses occurred later than the referenced time point. If an evoked event occurred later than the time in which the average response decayed back to baseline, it was used as the right-hand point on the integration window. In this approach, the time at which the earliest evoked rose to the time the latest evoked returned to baseline was used. This had to be determined first in all the data sets so a given window of time could be used throughout all the sweeps responding to that specific recording site.

Histograms of the evoked events were made for each 1,000 traces, and for any spontaneous event throughout the recording, to determine if shifts in peak and charge distributions occurred on the addition of 20-HE and 5-HT.

\section{RES ULTS}

\section{Regional differences of the opener muscle}

The anatomic arrangement of the ventral surface of the opener muscle in the first pair of walking legs is organized in a pinnate pattern, with the muscle fibers attached to the apodeme in the middle and to the cuticle on their sides. There is a small group of muscle fibers at the most proximal region the diameters of which are clearly different from the rest of these fibers (Fig. 1). The two regions in which the opener muscle commonly is divided for anatomic and physiological studies are the proximal and central regions due to their different regional features (Cooper et al. 1995a, 1996b,c; Iravani 1965). Although the entire opener muscle is innervated by only a single excitatory motor neuron, the preterminal branching and branch lengths are less on the proximal fibers than those of the central. Also, synapses of the varicose terminals have distinct differences both in gross

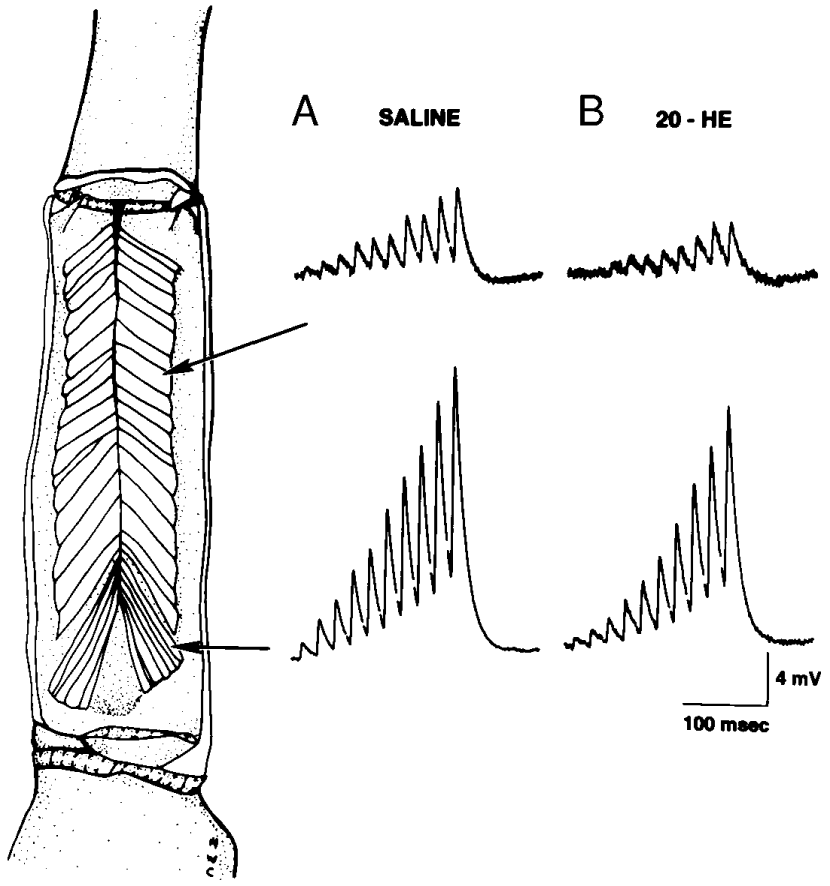

FIG. 1. Schematic of the opener muscle from a 1st walking leg showing the regional differences in synaptic response. $A$ : excitatory postsynaptic potentials (EPSPs) recorded with an intracellular electrode during a train of stimulation at $50 \mathrm{~Hz}(200-\mathrm{ms}$ duration) in crayfish saline. $B$ : reduction in amplitude of the EPSPs in the presence of 20-HE $(10 \mu \mathrm{M})$ after $20 \mathrm{~min}$. Delay between stimulus trains was $10 \mathrm{~s}$, and the traces shown are an average of 20 trials.

morphology and in synaptic structure. The terminals on the central muscle fibers are composed of long strings of varicosities, whereas the proximal region has shorter clusters of varicose terminals. Ultrastructurally, the proximal varicosities contain more synapses with multiple release sites, and this difference has been correlated directly to physiological recordings from individual varicosities (Cooper 1995a, 1996c). The differences in synaptic structures most likely contribute to the different presynaptic release properties (Cooper et al. 1996b), which in turn result in the varied postsynaptic responses measured in the two regions. A train of 10 stimuli applied at $50 \mathrm{~Hz}$ to the excitatory motor neuron while simultaneously recording intracellularly from a central and a proximal muscle fiber makes the differences in the EPSPs readily observable (Fig. 1).

\section{Effects of 20-HE on EPSP amplitude}

EPSPs gradually decrease on application of $10 \mu \mathrm{M} 20$ HE in saline. After $20 \mathrm{~min}$ of continuous bathing, the initial EPSPs of a train recorded centrally become greatly decreased even after an average of 20 trials (Fig. 1). The effect on the amplitude of the EPSPs is seen more easily in the recordings made from the proximal muscle fibers where the initial EPSP within a train is substantially larger (Fig. 1).

Facilitation measurements before and after the addition of 20-HE reveal a reduction in the transmission but not necessarily a reduction in the facilitation index $(\mathrm{Fe})$. There is no consistent trend in the $\mathrm{Fe}$ index in relation to 20-HE applica- 
TABLE 1. Effect of 20-HE on EPSP amplitude and facilitation

\begin{tabular}{|c|c|c|c|c|c|c|}
\hline & \multicolumn{3}{|c|}{ Initial EPSP amplitude, mV } & \multicolumn{3}{|c|}{$\mathrm{Fe}(10 \mathrm{th} / 1 \mathrm{st}-1)$} \\
\hline $\mathrm{C}$ & 0.25 & 0.18 & $\downarrow 28 \%$ & 7.5 & 8.54 & $\uparrow 13 \%$ \\
\hline $\mathrm{P}$ & 1.5 & 1.0 & $\downarrow 33 \%$ & 11.4 & 17.9 & $\uparrow 57 \%$ \\
\hline \multicolumn{7}{|c|}{ Preparation 2} \\
\hline C & 0.3 & 0.2 & $\downarrow 33 \%$ & 16.8 & 14.2 & $\downarrow 15 \%$ \\
\hline $\mathrm{C}$ & 0.19 & 0.12 & $\downarrow 37 \%$ & 8.2 & 21 & $\uparrow 156 \%$ \\
\hline $\mathrm{P}$ & 1.3 & 0.9 & $\downarrow 31 \%$ & 10.5 & 22 & $\uparrow 109 \%$ \\
\hline \multicolumn{7}{|c|}{ Preparation 4} \\
\hline C & 0.42 & 0.3 & $\downarrow 29 \%$ & 32 & 28 & $\downarrow 13 \%$ \\
\hline $\mathrm{P}$ & 2.34 & 1.4 & $\downarrow 40 \%$ & 11 & 10 & $\downarrow 9 \%$ \\
\hline \multicolumn{7}{|c|}{ Preparation 5} \\
\hline C & 0.37 & 0.21 & $\downarrow 43 \%$ & 51 & 46 & $\downarrow 9 \%$ \\
\hline
\end{tabular}

Repetitive measures in five preparations of excitatory postsynaptic potential (EPSP) initial amplitude and facilitation index (Fe) before and after 20 min of 20-hydroxyecdysone (20-HE) application. Each measure was taken for an average of 50 responses. The stimulation frequency was $50 \mathrm{~Hz}$ for 10 pulses (200-ms duration) with 10-s intervals between the trains. The Fe was measured by correcting the amplitude of the 10 pulse to baseline as discussed in METHODS. The mean \pm SE are tabulated at the bottom. * Comparing central $(C)$ and proximal $(\mathrm{P})$ responses there are significant differences $(P<$ 0.005 , Mann-Whitney) in the initial EPSP amplitudes. This is not the case for Fe index. $\ddagger$ In comparing the initial EPSP amplitude before and after the addition of 20-HE, there is a significant difference $(P<0.005$, Wilcoxon sign paired rank sum) to the reduction. There was not a significant change in the $\mathrm{Fe}$ index.

tion from preparation to preparation. This means that although 20-HE causes a reduction in the EPSP amplitude, the properties underlying facilitation may not be affected. The Fe index is notoriously difficult to obtain because of problems associated with correctly measuring the first EPSP amplitude within a train, due not only to the small size of the evoked response but also to a low signal-to-noise ratio. Various assessments to index facilitation measures are being sought (M. E. Crider and R. L. Cooper, unpublished data). For example, the ratio of the 10th to the 3rd EPSP ratio may prove more beneficial than the ratio of the 10th to the 1st EPSP. The initial EPSP amplitudes and Fe index for five opener muscle preparations measured in the central and proximal muscle fibers before and $20 \mathrm{~min}$ after the addition of 20-HE are listed in Table 1.

\section{Effects of 20-HE on quantal parameters}

Focal recordings made directly over visualized varicosities allow one to determine the quantal content for that location and to assess the effects on both the pre- and postsynaptic sites. The sites along the terminals on central and proximal fibers chosen for recording have been shown previously to exhibit a range of synaptic efficacies (Cooper et al. 1995a, 1996b). The representative sites used as examples of terminals in the central and proximal regions of the muscle are shown in Fig. 2. These terminals are traced from photographs of nerve terminals made at the time of placing the focal macropatch electrode on the varicosity of interest. The varicosity recorded is circled in the figures, the circles represent the rim of the macropatch electrode. The labeled terminals shown in Fig. 2 (i.e., $\mathrm{C}_{1}, \mathrm{C}_{2}, \mathrm{C}_{3}, \mathrm{P}_{1}$, and $\mathrm{P}_{2}$ ) are referred
A Central

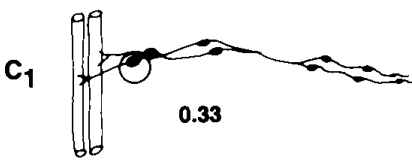

$\mathbf{P}_{1}$

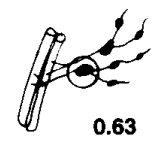

$c_{2}$

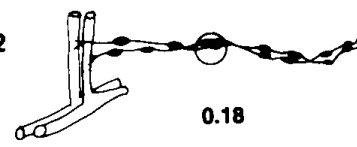

$\mathbf{P}_{\mathbf{2}}$

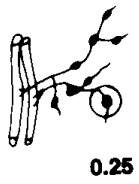

$c_{3}$

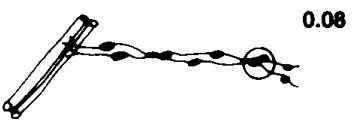

FIG. 2. Traces of the nerve terminals seen on central and proximal muscle fibers and the focal, macropatch recording sites are demarcated. Terminals are string-like on the central muscle fibers and more cluster-like on the proximal muscle fibers. These terminals were traced from photographs of the living nerve terminals visualized with the vital stain 4-Di-2Asp before placement of the focal electrode over the discretely identified varicosity. Outlines around a varicosity indicate the placement of the electrode. Mean quantal content by the direct counting method is given, which was obtained while the preparation was bathed in the initial saline solution. Scale bar, $10 \mu \mathrm{m}$. 


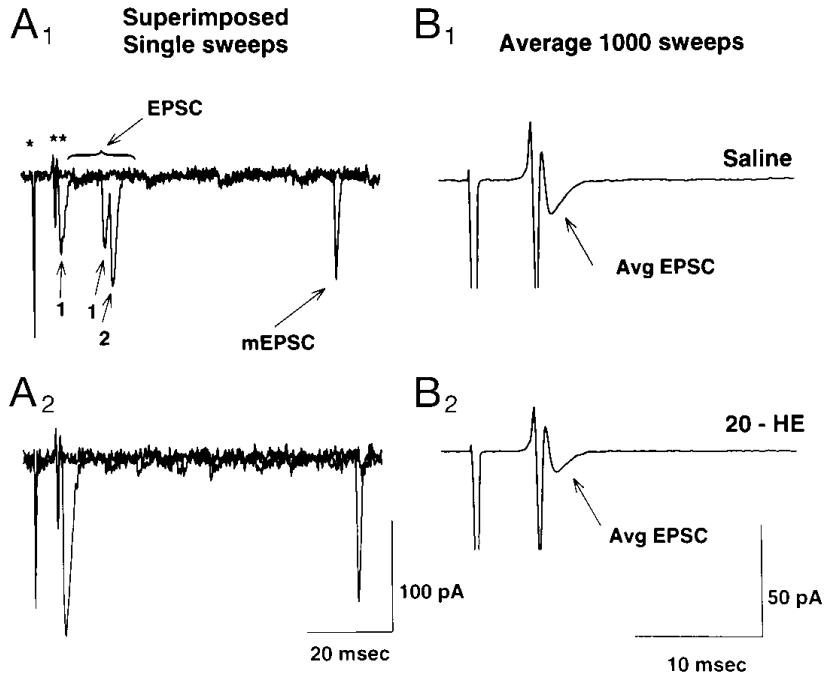

FIG. 3. Influence of 20-hydroxyecdysone (20-HE) on synaptic current as recorded with a focal macropatch electrode from a single visualized varicosity. Representative single traces in the presence of saline $(A l)$ and 20-HE (BI) are illustrated. Note in the evoked excitatory postsynaptic currents (EPSCs) individual quanta can be counted $(\rightarrow$ ). Occasional spontaneous release is shown that results in a miniature excitatory postsynaptic current (mEPSC). $\star$, stimulus artifact; $\star \star$, nerve terminal spike. $B$ : average of 1,000 trials in saline and after $20 \mathrm{~min}$ of bathing the preparation in 20HE. Stimulation frequency was at $1 \mathrm{~Hz}$.

to in Tables 3-5 and in other figures so that anatomic locations and functions may be correlated. Three string-like terminals on central fibers and two cluster-like terminals on proximal fibers are shown, each from different preparations. These structural differences among the terminals of the single motor neuron are typical for the central and proximal regions of the opener muscle (Cooper et al. 1995a, 1996ac). Individual macropatch recordings from a terminal are represented in Fig. 3. Single sweeps may contain a failure, an evoked response with one quantal event ( 1 of the traces in Fig. 3Al), or an evoked response with multiple releases (the superimposed single trace in Fig. 3Al). There are only two traces superimposed in each of the Figs. $3 A, 1$ and 2 . There may be a failure to elicit an evoked response as shown in one of the single traces of Fig. $3 A 2$.

In Fig. $3 A, 1$ and 2, spontaneous events occur much later than the nerve terminal spike. The mEPSCs indicate the unitary nature of the evoked events. There is no discernible difference in the distribution of the peak amplitude or area of the mEPSCs between the saline bath and to 20-HE treated preparations. Figure $3 A, 1$ and 2, represent general findings in that there are more failures in evoked EPSCs in the presence of 20-HE, although the spontaneous or mEPSCs still occur with relatively the same size and shape. The increase in the number of failures in the presence of 20-HE is apparent in the average of 1,000 traces and is shown in Fig. $3 B, I$ and 2 .

The gradual change in the peak amplitude of the EPSCs and the observed quantal occurrences are shown in Fig. 4. These responses indicate increased failures and fewer single and multiple evoked events. The direct method of counting quantal events alleviates difficulties associated with changes in the seal resistance as well as in the determination of the mean quantal content as determined by measuring either peak amplitude $\left(m_{\mathrm{pk}}\right)$ or charge $\left(m_{\mathrm{ch}}\right)$ of the responses. In experiments in which the seal resistance changed, only the direct counting method was used to determine the mean quantal content $\left(m_{\text {co }}\right)$ (Cooper et al. 1995b). This report includes all three methods of determination so that a comparative analysis could be done.

An advantage of directly counting evoked quanta is that one may make estimates regarding the quantal parameters based on the distribution of events, and further, on the effects that 20-HE has on these parameters. In Fig. $4, B$ and $C$, the increase in failures and reduction in the number of single events are shown with the $m_{\mathrm{co}}$ given after $20 \mathrm{~min}$ of bathing with 20-HE $(10 \mu \mathrm{M})$. To compare the gradual effects of 20HE on $m_{\mathrm{co}}, n$ (number of release sites), and $P$ (the average probability of release at the sites), the results from two representative experiments are shown in Fig. 5. The three quantal parameters $m_{\mathrm{co}}, n$, and $P$ are plotted over time for each set of 1,000 traces at a stimulation rate of $2.5 \mathrm{~Hz}$. The graph shows the long-term effect 20-HE has on reducing synaptic efficacy, even after the bathing solution is thoroughly exchanged with saline. In the presence of saline alone, the responses usually remain constant for a longer

A
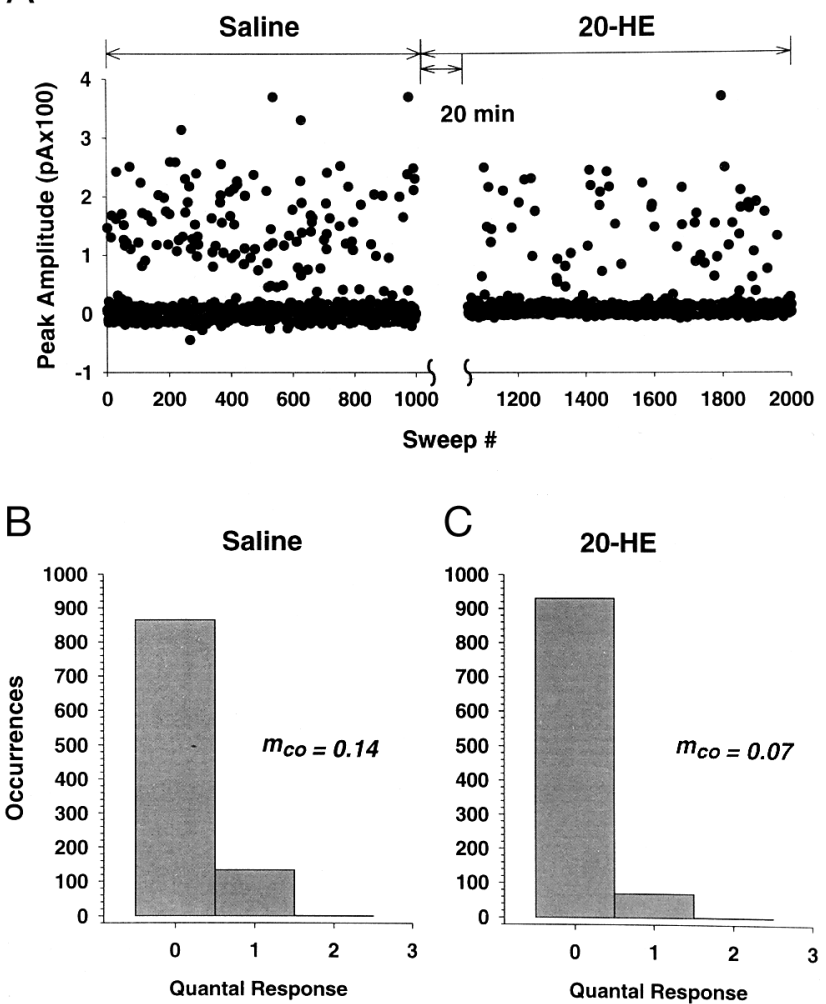

FIG. 4. Influence of 20-HE on evoked EPSC peak amplitude and on the number of individual quantal releases determined by direct counts is shown $A$ : initial 1,000 trials showed stationarity in the peak amplitude of the EPSC, but after the addition of 20-HE, the EPSC peak amplitude showed a substantial decrease. Peak amplitude was measured by taking the maximum peak of the evoked response within a given window of time. $B$ : direct counts of the evoked quantal events during the same 1,000 trials as shown in $A$ during the saline and 20-HE bathing times. Failures, 0; single events, 1; 2 events, 2 . 


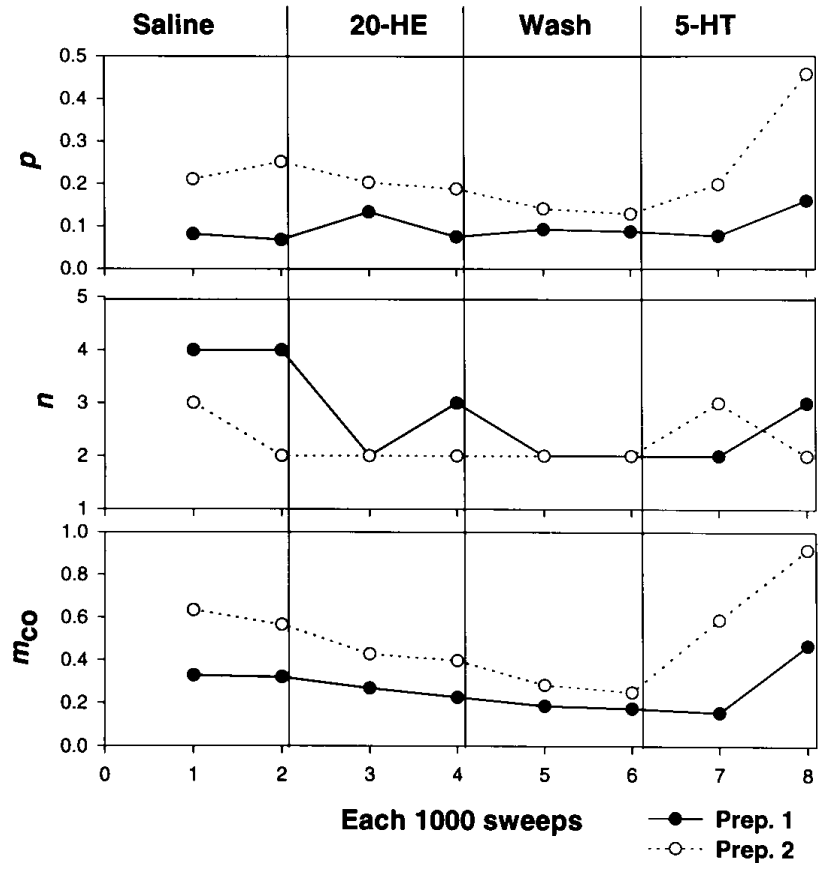

FIG. 5. Changes in the quantal parameters in 2 representative preparations as a result of the addition of 20-HE followed by a wash and serotonin (5HT) is illustrated. Mean quantal content $\left(m_{\text {co }}\right)$ determined by direct counts revealed a reduction in the presence of 20 -HE but a later increase with 5-HTcontaining bathing medium. Quantal parameters $n$ and $P$ were determined by the maximum likelihood estimation (MLE) as shown in Table 2.

time than the entire experimental protocol shown $(>8$ runs of a 1,000 traces each).

Because the synaptic responses continued to diminish after the application of 20-HE and washing, there was concern that the nerve terminal could not recover or enhance release, thus the bathing medium was changed to a crayfish saline containing $1 \mu \mathrm{M} 5-\mathrm{HT}$, a neuromodulator of crustaceans well known to cause an increase in synaptic transmission. The $m_{\text {co }}$ increases with 5-HT, resulting in a reversal of the decline induced by 20 -HE. The one-time exchange to 5HT bathing medium resulted in an upward trend for $\sim 20$ min before the quantal parameters leveled off, usually just above the baseline values.

To obtain estimates of the quantal parameters, each set of 1,000 trials was examined and analyzed to determine the statistical distribution that would best describe its profile. To obtain the $n$ and $P$ values and the standard errors of the estimation, this analysis was followed by a maximum likelihood estimation (Cooper et al. 1995b; Smith et al. 1991 ) and by bootstrapping procedures. The best distribution of the observed direct count data were found to be either binomial or Poisson in form. And if a binomial distribution was favored, then it was determined to be a uniform or nonuniform binomial distribution. The direct counts also allow the mean quantal content to be determined. The stepwise procedure is illustrated in Table 2 for a recording midway along the length of a string-like terminal on a central muscle fiber at $1-\mathrm{Hz}$ stimulation. This step-wise procedure was used to assess the quantal parameters for the recorded varicosities depicted in Fig. 2. The composite results are presented in Table 3, showing the effects of 20-HE followed by 5-HT on synaptic transmission. The percent change listed in the Table 3 for 20-HE was normalized for comparisons by taking the value determined in saline and subtracting the value calculated for the effects of 20-HE. The result then was divided by the saline value. The percent change in the parameters caused by the addition of 5-HT similarly was calculated. In each of the five locations recorded there was a substantial decrease in the mean quantal content $\left(m_{\text {co }}\right)$, along with a reduction in the probability $(P)$ of release and the number of release sites $(n)$. The later application of 5HT revived the mean quantal content in all cases along with substantial increases in $P$. In some cases ( 4 of 5 ) $n$ also increased (Table 3).

\section{Measure of EPSC amplitude and charge for determination of mean quantal content}

Two other procedures, besides the direct counts, were used to determine mean quantal content. They involved measuring the peak amplitude of evoked responses and spontaneous events. The mean quantal content was determined by dividing the averaged evoked response by the average of the spontaneous events. A third procedure was used to calculate the area or charge $(\mathrm{pA} \times \mathrm{ms})$ under the curve for evoked and spontaneous measurements. The measurement of evoked peak amplitude frequently is obtained from the maximum value of all the averaged traces. This type of measure is the same as taking a fixed point in time and measuring each individual evoked response relative to the fixed point to obtain an average value. Unfortunately, this type of measure severely underestimates the true evoked response from the nerve when latency jitter in release occurs (Cooper et al. 1995b). As shown in Fig. 3 for individual traces, substantial latency jitter is present in the evoked responses, and the complication of multiple release is underestimated. To circumvent this issue, a window of time representing the evoked response time is established. This allows the maximum of any response within the window to be measured. Obtaining the averaged evoked peak amplitude with the time variable always has provided a larger mean quantal content ( $m_{\text {peak }}$ variable) in these studies (Table 4$)$. The percent difference in these two methods is presented and clearly indicates the need to consider the latency jitter of release in the opener motor nerve terminal. For comparison purposes, the mean quantal content for the charge measurement method and direct counts also are presented in Table 4 . The measurement of charge also was obtained by setting a time window to measure any response that occurred within a set time so that latency jitter would not be a concern (Borst and Sakmann 1996). These procedures have been described in detail (Cooper et al. 1995b).

The distributions presented in the histogram of Fig. 6 exemplify the differences among the ways to measure peak amplitude (variable time) and charge of evoked and spontaneous events. The effects of 20-HE in the two types of measurement are different; the number of larger and smaller values is decreased, and the number of failures is increased. The distribution of spontaneous events is not shifted as a result of 20-HE application (Fig. 6, insets). To determine 
TABLE 2. Direct counts of quantal events and estimates of quantal parameters $n, P$ in the presences of 20-HE and 5-HT

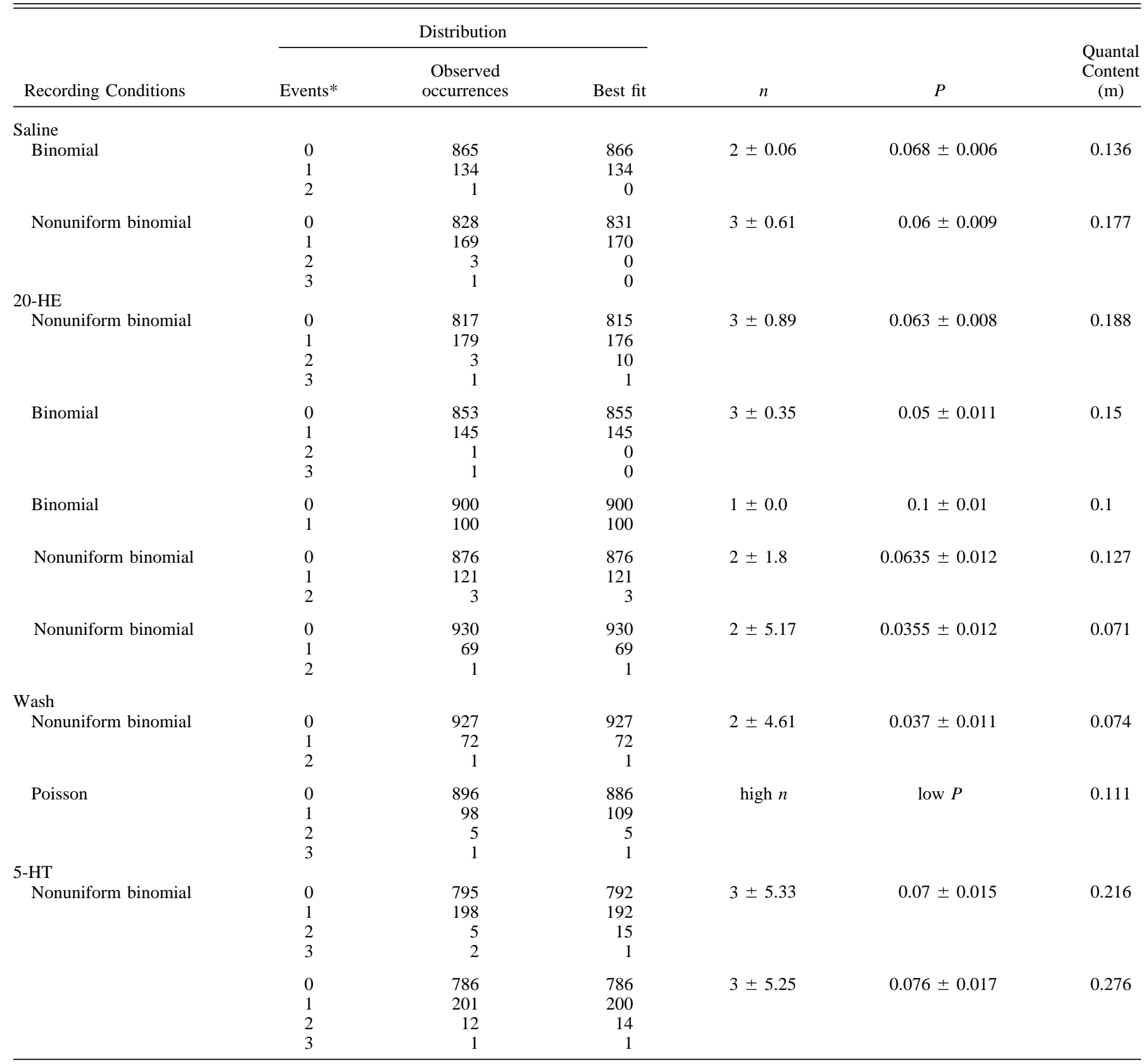

The time series of collecting groups of 1,000 trials is represented in the order in which the experiment was performed. The distributions shown were obtained from the best fit distribution. The type of distribution the observed events best fit to are included. $n$ and $P$ values are in means \pm SE as determined by the maximum likelihood estimation (MLE) and bootstrapping procedures (see Cooper et al., 1995b; Efron and Tibshirani, 1993). * Number of discrete events indicated as: 0 , failures; 1 , ones; 2 , twos; etc.

if this is the case for all recorded sites, the means in the distribution of the spontaneous events measured by peak amplitude and charge were calculated along with the coefficient of variation (CV). Table 5 shows that indeed neither the size nor the overall charge is altered substantially by the application of 20-HE. To better illustrate the individual responses when the preparation is bathed in saline, when treated with 20-HE, during the washout of 20-HE, and after the application of 5-HT, the values for peak amplitude and charge are shown in Fig. 7. This is a representative case except that there was a longer time of exposure to 20-HE than in prior experiments. The longer bathing time in 20HE for this particular experiment was used to determine if increased exposure to a constant concentration of $20-\mathrm{HE}$ would result in any measurable effect on the spontaneous events. It did not.

\section{I S C USSION}

The results presented in this study indicate that the probability of vesicular release at active motor nerve terminals is 
TABLE 3. Percent change in quantal parameter with the addition 20-HE and 5-HT

\begin{tabular}{|c|c|c|c|c|c|c|c|c|}
\hline Site & $\% \Delta m_{\mathrm{CO}}$ & $\% \Delta P$ & $n$ & Distribution & $\% \Delta m_{\mathrm{CO}}$ & $\% \Delta P$ & $n$ & Distribution \\
\hline $\mathrm{C}_{2}$ & $\downarrow 48$ & $\downarrow 48$ & $3 \rightarrow 2$ & NUB to $\mathrm{B}$ & $\uparrow 288$ & $\uparrow 114$ & $2 \rightarrow 3$ & $\mathrm{~B}$ to NUB \\
\hline $\mathrm{C}_{3}$ & $\downarrow 28$ & $\downarrow 48$ & $2 \rightarrow 3$ & NUB to NUB & $\uparrow 114$ & $\uparrow 221$ & $3 \rightarrow 2$ & NUB to NUB \\
\hline $\mathrm{P}_{1}$ & $\downarrow 55$ & $\downarrow 33$ & $3 \rightarrow 2$ & NUB to NUB & $\uparrow 226$ & $\uparrow 224$ & $2 \rightarrow 3$ & NUB to NUB \\
\hline $\mathrm{P}_{2}$ & $\downarrow 39$ & $\downarrow 8$ & $3 \rightarrow 2$ & NUB to NUB & $\uparrow 200$ & $\uparrow 71$ & $2 \rightarrow 3$ & NUB to NUB \\
\hline
\end{tabular}

The quantal parameters were calculated from the 1,000 sweeps proceeding the addition of 20-HE in saline to compare with the 1,000 sweeps after 20 min of exposure to 20-HE. The effect of adding 5-HT were determined in the first 100 trials upon addition of 5-HT in comparison with the proceeding 1,000 sweeps in 20-HE. Mean quantal content $\left(m_{\mathrm{CO}}\right)$ was determined by direct counts. Depending on the type of distribution that best fit the observed counts as shown in Table 2, the probability of release $(P)$ was estimated by the MLE method. The distributions were either binomial (B) or nonuniform binomial (NUB).

decreased by the steroid molting hormone, 20-HE. The effect of 20-HE on neuromuscular junctions of intermolt crayfish is too rapid to be accounted for by steroid receptor effects on gene expression; more likely the effect is exerted directly on nerve terminals. The consequences of fewer vesicles being released are smaller evoked postsynaptic currents, which in turn produces smaller EPSPs across the muscle fiber membrane. In a nonspiking muscle fiber, graded reduction in EPSPs reduces the potential change across the entire fiber, thus weakening the contraction; 20-HE reduces the force of muscle contraction.

When crayfish prepare to molt, the inner cuticular layer is soft, and powerful muscle contraction can easily damage the animal by separating the inner and outer layers of the cuticle. Immediately after a molt, the cuticle remains soft for a time and vulnerable to intense muscular contraction.

TABLE 4. Mean quantal content determined by four approaches

\begin{tabular}{lllllll} 
& & \multicolumn{2}{c}{$m_{\text {peak }}$} & & & \\
\cline { 3 - 4 } Site & Solution & Variable & Fixed & $\% \Delta$ & $m_{\text {charge }}$ & $m_{\text {count }}$ \\
\hline $\mathrm{C}_{1}$ & Saline & 0.454 & 0.364 & $\downarrow 20 \%$ & 0.322 & 0.327 \\
& 20-HE & 0.310 & 0.218 & $\downarrow 30 \%$ & 0.197 & 0.185 \\
$\mathrm{C}_{2}$ & Saline & 0.179 & 0.154 & $\downarrow 14 \%$ & 0.168 & 0.177 \\
& 20-HE & 0.122 & 0.107 & $\downarrow 12 \%$ & 0.045 & 0.071 \\
$\mathrm{C}_{3}$ & Saline & 0.047 & 0.0095 & $\downarrow 80 \%$ & 0.077 & 0.079 \\
& 20-HE & 0.032 & 0.003 & $\downarrow 91 \%$ & 0.049 & 0.057 \\
$\mathrm{P}_{1}$ & Saline & 0.850 & 0.700 & $\downarrow 18 \%$ & 0.820 & 0.633 \\
& 20-HE & 0.418 & 0.143 & $\downarrow 66 \%$ & 0.328 & 0.283 \\
$\mathrm{P}_{2}$ & Saline & 0.192 & 0.054 & $\downarrow 72 \%$ & 0.189 & 0.251 \\
& 20-HE & 0.021 & 0.005 & $\downarrow 76 \%$ & 0.044 & 0.154 \\
\hline
\end{tabular}

The mean quantal content determined by measuring the maximum evoked peak amplitude ( $m_{\text {peak }}$ fixed) of the average of all sweeps compared with the maximum evoked peak amplitude ( $m_{\text {peak }}$ variable) within each sweep reveals that there is latency jitter in the saline groups as well as in the 20HE groups. The mean quantal content determined by measuring evoked charge ( $\left.m_{\text {charge }}\right)$ is shown along with the direct method of counting evoked quantal events $\left(m_{\text {count }}\right)$. The sites correspond to the ones shown in Fig. 4. The $m_{\text {peak }}$ fixed are significantly lower in value than $m_{\text {peak }}$ variable and $m_{\text {charge }}$ $(P<0.005$, Wilcoxon sign paired rank sum $)$, which demonstrates the problematic nature of latency jitter. The $m_{\text {peak }}$ fixed are significantly lower in value than $m_{\text {peak }}$ variable and $m_{\text {charge }}(P<0.005$, Wilcoxon sign paired rank sum), which demonstrates the problematic nature of latency jitter. The $m_{\text {charge }}$ showed significant difference to the $m_{\text {count }}(P<0.05$, Wilcoxon sign paired rank sum), and $m_{\text {count }}$ is significantly lower than $m_{\text {peak }}$ variable $(P<$ 0.05 , Wilcoxon sign paired rank sum).
The effect of 20-HE thus appears to constitute at least part of a compensatory mechanism to reduce injury.

The measurable neurophysiological parameters fit well with such injury avoidance behavior of arthropods. Adult Cancer magister crabs become very sedentary during the few days preceding a molt and will bury themselves in sand in the bottom of a holding tank if given the opportunity (personal observations). The same behavior occurs in the first few days after a molt until the shell hardens. It is not known if this same behavior is seen in their natural environment. Lobsters reduce muscle activity by reducing repetitive episodes of escape swimming (Cromarty et al. 1991). Molting insects reduce the use of locomotive muscles until pupation. These behaviors may be due in part to reduced synaptic efficacy at neuromuscular junctions as a direct effect of 20HE. It remains to be determined if there are effects on central and peripheral sensory systems that also might play roles in modifying behavior. The effect of 20-HE on primary sensory systems, and the integration of sensory input in the crayfish is currently under investigation ( $\mathrm{Li}$ et al. 1997).

In lobsters, the establishment of a dominant and submissive status within a pair is believed to be due to the levels of the neuromodulators serotonin and octopamine (Kravitz 1988, 1990; Kravitz et al. 1980). Directly measuring circulating levels of modulators related to behavioral status would be an excellent way to confirm such a theory. Knowing the level of one particular neuromodulator may be misleading, though, as it is likely that it is the ratios of such neuromodulators that drive these responses. A delicate balance of hormone levels is of great importance in regulating physiological function throughout the animal kingdom. For a review of modulator effects of multiple hormones in insects, refer to Steel and Davey (1985); and in the same review volume, refer to Riddford (1985) on the levels of ecdysteroids related to various cellular functions.

It is of particular importance to know the molt stage of crustaceans when monitoring behavior as well as when conducting neurophysiological experimentation. For example, the depression in synaptic transmission caused by 20 -HE can likely cause target tissue to be more, or possibly even less, responsive to 5-HT. These possibilities were tested by the application of 5-HT after the neuromuscular junction was depressed by 20-HE. Synaptic efficacy was enhanced substantially by 5 -HT in the absence of prior treatment with 


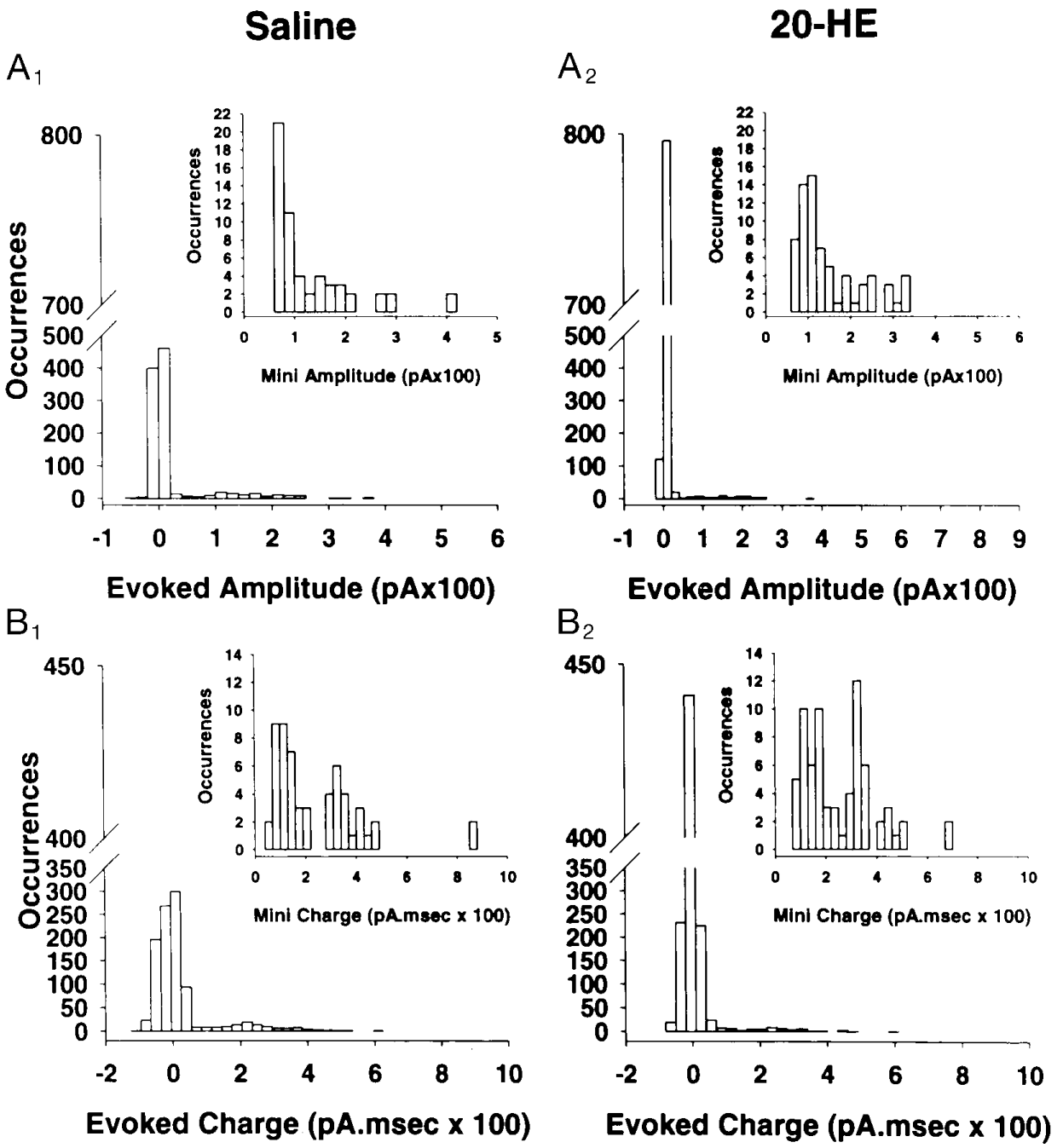

FIG. 6. Distribution profiles are shown of evoked and spontaneous releases measured before $(A I$ and $B I)$ and during the application of 20-HE ( $A 2$ and $B 2)$. Evoked release shows a reduction in the peak amplitude and charge, whereas the spontaneous events (insets) do not indicate an alteration in their distributions.
20-HE. But when 20-HE was applied to the preparation followed by a dose of 5-HT, the same degree of synaptic enhancement was not present. Because it has been shown that 5-HT works through $\mathrm{IP}_{3}$ to increase neurotransmission in the same preparation as used in this study (Delaney et al. 1991; Dixon and Atwood 1989), it is likely that 20-HE is using a different signalling pathway to produce its effects. On the other hand, if $20-\mathrm{HE}$ is working as a negative influence on the $\mathrm{IP}_{3}$ cascade, 5-HT can override its effect to produce a slight enhancement of transmission. The quickacting effect of 5-HT released into hemolymph could be the ideal candidate to allow a premolting animal, with high levels of 20-HE and depressed motor terminals, to escape from a predator or to defend itself over a short period of time.

Documented mechanisms of nongenomic effects of sex steroids result in rapid responses that often can be modified later by the long-acting genomic actions of the same steroid hormones (Tuohimaa et al. 1996). Only the rapid effects of 20-HE are addressed in this paper. The genomic effects on motor neurons and muscle fibers remain unaddressed. The effects presented here are considered to be nongenomic not only because they occur in an isolated leg with the motor axon severed from its cell body but also because the effects appear within a few minutes of switching the bathing medium to one containing 20-HE. From the quantal analysis of synaptic transmission, these rapid effects appear to be due entirely to alterations in the release process at the presynaptic terminals. Possible long-term effects on muscle fibers may additionally alter the responsiveness to neurotransmitter and neuromodulators, but this remains to be determined. Because a number of muscles are known to degrade before molting, their responsiveness to chemical transmission and contraction is likely to be altered (Mykles and Skinner 1982). Questions concerning the physiological state of a muscle during a molt and the altered behaviors, as well as responsiveness to neuromodulators, remain unanswered.

When the preparation is bathed with 20-HE, the effect begins within a few minutes and transmission continues to decrease for $\leq 1 \mathrm{~h}$. The effect cannot be reversed by simply exchanging the bathing medium and then washing the preparation thoroughly with $20-\mathrm{HE}-$ free saline. The addition of 5 -HT or an increase the stimulus frequency can offset the inhibitory influence of $20-\mathrm{HE}$ on the nerve terminal. Because the spontaneous events do not show any significant change or trend in decreasing the size or the charge, 20-HE does not affect the postsynaptic glutamate receptors in these neu- 
TABLE 5. Mean peak amplitudes and charge of the spontaneous events recorded before and after 20 min of exposure to $20-H E$

\begin{tabular}{|c|c|c|c|c|c|c|}
\hline \multirow[b]{2}{*}{ Site } & \multirow[b]{2}{*}{ Solution } & \multicolumn{2}{|c|}{ Peak $(\mathrm{pA} \times 100)$} & \multicolumn{2}{|c|}{$\begin{array}{c}\text { Charge } \\
(\mathrm{pA} \cdot \mathrm{ms} \times 100)\end{array}$} & \multirow{2}{*}{$\begin{array}{l}\text { Frequency of } \\
\text { mEPSCs, Hz }\end{array}$} \\
\hline & & Mean & $\mathrm{CV}$ & Mean & $\mathrm{CV}$ & \\
\hline \multirow[t]{2}{*}{$\mathrm{C}_{1}$} & Saline & $1.008(32)$ & 60 & $2.265(32)$ & 87 & 0.14 \\
\hline & 20-HE & $1.481(28)$ & 62 & $2.915(28)$ & 62 & 0.62 \\
\hline \multirow[t]{2}{*}{$\mathrm{C}_{2}$} & Saline & $1.091(28)$ & 76 & $2.036(28)$ & 86 & 0.11 \\
\hline & 20-HE & $1.276(35)$ & 60 & $2.252(35)$ & 61 & 0.07 \\
\hline \multirow[t]{2}{*}{$\mathrm{C}_{3}$} & Saline & $1.337(100)$ & 50 & $2.316(100)$ & 70 & 0.5 \\
\hline & 20-HE & 1.322 (193) & 45 & 2.409 (193) & 88 & 0.64 \\
\hline \multirow[t]{2}{*}{$\mathrm{P}_{1}$} & Saline & $1.557(22)$ & 47 & $4.1(22)$ & 77 & 0.14 \\
\hline & 20-HE & $1.010(21)$ & 49 & $3.329(21)$ & 75 & 0.117 \\
\hline \multirow[t]{2}{*}{$\mathrm{P}_{2}$} & Saline & $1.080(41)$ & 52 & $3.952(41)$ & 82 & 0.41 \\
\hline & 20-HE & $1.642(52)$ & 37 & $4.387(52)$ & 71 & 0.26 \\
\hline
\end{tabular}

The means of the distributions are presented along with the coefficient of variation $(\mathrm{CV})$ for each recording period. The sites correspond to those shown in Fig. 4. There is no significant difference among the peak amplitudes or the charge of the spontaneous events before and after the addition of 20-HE. The average frequency of spontaneous events during the sampling period has a trend to decrease upon application of $20-\mathrm{HE}$, although it is not significant due to small sample size. $n$ values are in parentheses.

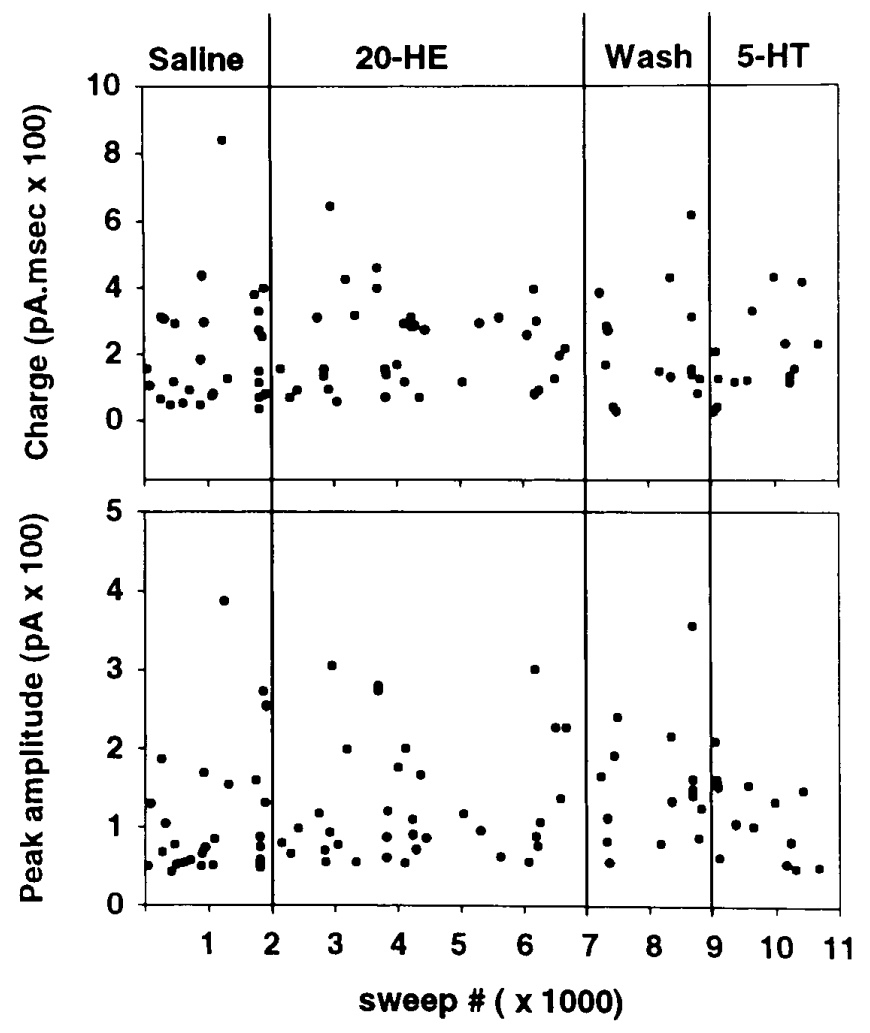

FIG. 7. Typical representation is shown of the peak amplitude and charge of spontaneous events while the preparation is bathed in crayfish saline, 20-HE for a prolonged time followed by a brief wash and addition of 5-HT. Stimulation frequency was at $1 \mathrm{~Hz}$, and the duration of a individual sweep was $500 \mathrm{~ms}$. Note that there is no trend of shifting the size or charge distributions by the various treatments. romuscular junctions. These results indicate a presynaptic effect, and possible sites of action are numerous. For example, 20-HE may bind to presynaptic glutamate autoreceptors, altering feedback control of the release process. These receptors have been shown to be metabotropic and present on the crustacean nerve terminals (Miwa et al. 1990). If there is an ecdysone receptor on the nerve terminal that influences one of the many signalling cascades, the effects would likely be seen on various processes such as vesicle docking, fusion, or recycling. Other possible actions could be directly on the voltage-gated sodium or calcium channels, allowing less calcium influx during a stimulus. The mechanism of action on the presynaptic terminals needs to be investigated to understand the role of $20-\mathrm{HE}$ in decreasing synaptic efficacy. Evidence is presented that narrows the site of 20-HE's action to the presynaptic terminal in reducing vesicular release.

We thank A. Cooper, Dr. Phil Bonner, and J. Wegrzyn for editorial assistance and critical comments. Illustrations were provided by H. W. Cooper. Appreciation is given to Dr. Phil Bonner for the use of microscopy equipment and a collegial atmosphere, which allowed this work to proceed. We thank Dr. John G. Nicholls (Basel) for endless encouragement and enthusiastic support and for first teaching us the uses of macropatch recordings.

Funding was provided by the University of Kentucky Research and Graduate Studies (R. L. Cooper) and Howard Hughes Medical Institute undergraduate training fellowship (M. E. Ruffner).

Address reprint requests to R. L. Cooper.

Received 4 September 1997; accepted in final form 11 December 1997.

\section{REFERENCES}

Borst, J.G.G. AND SAKMANN, B. Calcium influx and transmitter release in a fast CNS synapse. Nature 383: 431-434, 1996.

Cooper, R. L., Hampson, D., And Atwood, H. L. Synaptotagmin-like expression in the motor nerve terminals of crayfish. Brain Res. 703: $214-$ 216, 1996a.

Cooper, R. L., Harrington, C. C., Marin, L., And Atwood, H. L. Quantal release at visualized terminals of a crayfish motor axon: intraterminal and regional differences. J. Comp. Neurol. 375: 583-600, 1996 b.

Cooper, R. L., Marin, L., AND Atwood, H. L. Synaptic differentiation of a single motor neuron: conjoint definition of transmitter release, presynaptic calcium signals, and ultrastructure. J. Neurosci. 15: 4209-4222, 1995a.

Cooper, R. L., Stewart, B. A., Wojtowicz, J. M., Wang, S., And AtwOOD, H. L. Quantal measurement and analysis methods compared for crayfish and Drosophila neuromuscular junctions and rat hippocampus. J. Neurosci. Methods 61: 67-79, 1995b.

Cooper, R. L., Winslow, J., Govind, C. K., And Atwood, H. L. Synaptic structural complexity as a factor enhancing probability of calcium-mediated transmitter release. J. Neurophysiol. 75: 2451-2466, 1996c.

Crider, M. E. AND CoOPER, R. L. Modulation of transmitter release by 5-HT at phasic and tonic neuromuscular junctions in crayfish: quantal parameters assessed. Soc. Neurosci. Abstr. 22: 309.13, 1996.

CRIDER, M. E. AND CoOper, R. L. The effects of 5-HT and octopamine as dual neuromodulators at the crayfish neuromuscular junction. Soc. Neurosci. Abstr. 23: 148.7, 1997.

Cromarty, S. I. Neuroethology of the Escape Behavior in the American Lobster, Homarus americanus, Over the Molt Cycle ( $\mathrm{PhD}$ dissertation). Kingston, RI: Univ. of Rhode Island, 1995.

Cromarty, S. I., Cobb, J. S., And Kass-Simon, G. Behavioral analysis of the escape response in the juvenile lobster Homarus americanus over the molt cycle. J. Exp. Biol. 158: 565-581, 1991.

Delaney, K., TAnK, D. W., AND Zucker, R. S. Presynaptic calcium and serotonin-mediated enhancement of transmitter release at crayfish neuromuscular junction. J. Neurosci. 11: 2631-2643, 1991.

Del Castillo, J. and Katz, B. Quantal components of the end-plate potential. J. Physiol. (Lond.) 124: 560-573, 1954.

Dixon, D. AND ATwood, H. L. Conjoint action of phosphoinositol and 
adenylate cyclase systems in serotonin-induced facilitation at the crayfish neuromuscular junction. J. Neurophysiol. 62: 1251-1259, 1989.

Dudel, J. AND KuFFLER, S. W. The quantal nature of transmission and spontaneous miniature potentials at the crayfish neuromuscular junction. J. Physiol. (Lond.) 155: 514-529, 1961.

EFRON, B. AND TIBSHIRANI, R. The bootstrap estimate of standard error. In: An Introduction to the Bootstrap, edited by B. Efron and R. Tibshirani. New York: Chapman Hall, 1993, p. 514-529.

IRAVANI, J. Membrandepolarisation der muskelfasern des öffnermuskels des flusskrebses auf nervenreiz und kaliumapplikation. Experientia 21: 609$610,1965$.

JACoBs, G. A. AND Weeks, J. C. Postsynaptic changes at a sensory-tomotorneuron synapse contribute to the developmental loss of a reflex behavior during insect metamorphosis. J. Neurosci. 10: 1341-1356, 1990.

Johnson, E. W. AND WeRnig, A. The binomial nature of transmitter release at the crayfish neuromuscular junction. J. Physiol. (Lond.) 218: $757-$ 767, 1971.

KRAVITZ, E. A. Hormonal control of behavior: amines and biasing behavioral output in lobsters. Science 241: 1775-1781, 1988

KRAVITZ, E. A. Hormonal control of behavior: amines as gain-setting elements that bias behavioral output in lobsters. Am. Zool. 30: 595-608, 1990.

Kravitz, E. A., Glusman, S., Harris-Warrick, R. M., Livingstone, M. S., SCHWARZ, T., AND GoY, M. F. Amines and a peptide as neurohormones in lobsters: actions on neuromuscular preparations and preliminary behavioral studies. J. Exp. Biol. 89: 159-175, 1980.

Levine, R. B., Truman, J. W., Linn, D., and Bate, C. M. Endorcrine regulation of the form and function of axonal arbors during insect metamorphosis. J. Neurosci. 6: 293-299, 1986.

LEVINE, R. B. AND WeEKS, J. C. Cell culture approaches to understanding the actions of steroid hormones on the insect nervous system. Dev. Neurosci. 18: 73-86, 1996.

Li, H., Ward, E., BRADACS, H., AND COOPER, R. L. Neuromodulator effects on primary sensory neurons: rapidly and slowly adapting proprioceptors. Soc. Neurosci. Abstr. 23: 313.8, 1997.

Magrassi, L., Purves, D., And Lichtman, J. W. Fluorescent probes that stain living nerve terminals. J. Neurosci. 7: 1207-1214, 1987.

Martin, A. R. A further study of the statistical composition of the endplate potential. J. Physiol. (Lond.) 130: 114-122, 1955.

MARTIN, A. R. The effect of membrane capacitance on non-linear summation of synaptic potentials. J. Theor. Biol. 59: 179-187, 1976.
Miwa, A., Ui, M., AND KaWAI, N. G protein is coupled to presynaptic glutamate and GABA receptors in lobster neuromuscular synapse. $J$. Neurophysiol. 63: 173-180, 1990.

MYKLES D. L. AND SKINNER D. M. Molt-cycle associated changes in calcium-activated proteinase activity that degrades actin and myosin in crustacean muscle. Dev. Biol. 92: 386-397, 1982.

RIDDFORD, L. M. Hormone action at the cellular level. In: Comprehensive Insect Physiology Biochemistry and Pharmacology, edited by G. A. Kerkut and L. I. Gilbert. New York: Pergamon Press, 1985, vol. 8, p. 3784.

Segraves, W. A. Steroid receptors and other transcription factors in ecdysone response. Recent Prog. Horm. Res. 49: 167-195, 1994.

Sмith, B. R., Wojtowicz, J. M., And Atwood, H. L. Maximum likelihood estimation of nonuniform transmitter release propabilities at the crayfish neuromuscular junction. J. Theor. Biol. 150: 457-472, 1991.

Steel, C.G.H. And Davey, K. G. Integration in the insect endocrine system. In: Comprehensive Insect Physiology Biochemistry and Pharmacology, edited by G. A. Kerkut and L. I. Gilbert. New York: Pergamon Press, 1985, vol. 8, p. 1-36.

Tuohimaa, P., Blauer, M., Pasanen, S., Passinen, S., Pekki, A., Punnonen, R., Syvala, H., Valkila, J., Wallen, M., Valiaho, J., Zhuang, Y., AND YLiKomi, T. Mechanisms of action of sex steriod hormones: basic concepts and clinical correlations. Maturitas 23, Suppl.: S3-S12, 1996.

Thummel, C. S. Flies on steroids-Drosophila metamorphosis and the mechanisms of steroid hormone action. Trends Genet. 12: 306-310, 1996.

Truman, J. W. Steroid receptors and nervous system metamorphosis in insects. Dev. Neurosci. 18: 87-101, 1996.

Truman, J. W. AND ReIss, S. W. Neuromuscular metamorphosis in the moth Manduca sexta: hormonal regulation of synapse loss and remodeling. J. Neurosci. 15: 4815-4826, 1995.

WeHLING, M. Looking beyond the dogma of genomic steroid action: insights and facts of the 1990s. J. Mol. Med. 73: 439-447, 1995

WERNIG, A. Estimates of statistical release parameters from crayfish and frog neuromuscular junctions. J. Physiol. (Lond.) 244: 208-221, 1975.

White, K. P., Hurban, P., Watanabe, T., and Hogness, D. S. Coordination of Drosophlia metamorphosis by two ecdysone-induced nuclear receptors. Science 276: 114-117, 1997.

Wojtowicz, J. M., Smith, B. R., And Atwood, H. L. Activity-dependent recruitment of silent synapses. Ann. NY Acad. Sci. 627: 169-179, 1991. 